\title{
Erratum
}

\section{Dynamic theory of quasilinear parabolic systems}

\section{Global existence}

\section{Herbert Amann}

Mathematisches Institut, Universität Zürich, Rämistrasse 74, CH-8001 Zürich, Switzerland

Math. Z. 202, 219-250(1989)

In one of the main results of that paper, namely in Theorem 2.1 , one has to add the following hypothesis: $\left(\mathscr{A}_{\pi}\left(m\left(x_{0}\right)\right), \mathscr{B}_{\pi}\left(m\left(x_{0}\right)\right)\right)$ is normally elliptic for each $x_{0} \in \bar{\Omega}$.

This assumption guarantees the validity of (2.1) which does not seem to follow - as we claimed - from the fact that $(\mathscr{A}(\mathrm{m}), \mathscr{B}(\mathrm{m}))$ is normally elliptic. It should be noted, however, that this additional assumption is satisfied in all concrete instances of normally elliptic systems, in particular, in the cases studied in the remainder of the paper.

In addition, the proof of Corollary 1.2 contains a gap. If $2 \alpha=1$, one has to use the following estimate:

$$
\begin{aligned}
\left|\int_{\Omega}\left\langle v, c_{j} \partial_{j} u\right\rangle d x\right| & \leqq \int_{\Omega}\left|\left\langle v,\left(c_{j}-c_{j}^{\varepsilon}\right) \partial_{j} u\right\rangle\right| d x+\int_{\Omega}\left|\left\langle v, c_{j}^{\varepsilon} \partial_{j} u\right\rangle\right| d x \\
& \leqq c\|v\|_{1 / 2} \sum_{j}\left(\left\|c_{j}-c_{j}^{\varepsilon}\right\|_{p_{1}}\|u\|_{1 / 2}+\left\|c_{j}^{\varepsilon}\right\|_{C^{1}}\|u\|_{\beta}\right),
\end{aligned}
$$

where $\beta<1 / 2$ and $c_{j}^{\varepsilon} \in C^{1}(\bar{\Omega}, X)$ are chosen so that

$$
\sum_{j}\left\|c_{j}-c_{j}^{\varepsilon}\right\|_{p_{1}}<\varepsilon / 2 c
$$

Now we obtain the assertion by estimating $\|u\|_{\beta}$ by means of (1.2).

Similarly, in (2.3) one has to replace $m$ by $m-m\left(x_{l}\right)$. Consequently, (2.6) and (2.7) have to be replaced by

$$
\left\langle v,\left(\lambda+A\left(m\left(x_{l}\right)\right)\right) \varphi_{l} u\right\rangle=\left\langle v, \varphi_{l}(\lambda+A(m)) u\right\rangle+R_{l}
$$

and

$$
R_{l}:=\left\langle v,\left(A\left(m\left(x_{l}\right)\right)-A\right)\left(\varphi_{l} u\right)\right\rangle+\left\langle v, A\left(\varphi_{l} u\right)-\varphi_{l} A u\right\rangle,
$$

respectively. Now the proof of Theorem 2.1 goes through along the indicated lines.

Lastly, it should be noted that the assumption $2 \alpha \geqq 1$ can be replaced by $1 / p<2 \alpha<1+1 / p$. 